\title{
N-state random switching based on quantum tunnelling
}

\author{
Ramón Bernardo Gavito ${ }^{\mathrm{a},},{ }^{*}$, Fernando Jiménez Urbanos ${ }^{\mathrm{b}}$, Jonathan Roberts ${ }^{\mathrm{a}}$, James Sexton $^{\mathrm{c}}$, \\ Benjamin Astbury ${ }^{\mathrm{a}}$, Hamzah Shokeir ${ }^{\mathrm{a}}$, Thomas McGrath ${ }^{\mathrm{a}}$, Yasir J. Noori ${ }^{\mathrm{a}}$, Christopher S. \\ Woodhead ${ }^{\mathrm{a}}$, Mohamed Missous ${ }^{\mathrm{c}}$, Utz Roedig ${ }^{\mathrm{a}}$, Robert J. Young ${ }^{\mathrm{a}}$ \\ ${ }^{a}$ Lancaster University, LA1 4YW, Lancaster, UK; ' IMDEA Nanoscience, Faraday 9, 28049, \\ Madrid, Spain; ${ }^{\mathrm{C}}$ The University of Manchester, M13 9PL, Manchester, UK \\ *r.bernardogavito1@lancaster.ac.uk
}

\begin{abstract}
In this work, we show how the hysteretic behaviour of resonant tunnelling diodes (RTDs) can be exploited for new functionalities. In particular, the RTDs exhibit a stochastic 2-state switching mechanism that could be useful for random number generation and cryptographic applications. This behaviour can be scaled to N-bit switching, by connecting various RTDs in series. The InGaAs/AlAs RTDs used in our experiments display very sharp negative differential resistance (NDR) peaks at room temperature which show hysteresis cycles that, rather than having a fixed switching threshold, show a probability distribution about a central value. We propose to use this intrinsic uncertainty emerging from the quantum nature of the RTDs as a source of randomness. We show that a combination of two RTDs in series results in devices with three-state outputs and discuss the possibility of scaling to N-state devices by subsequent series connections of RTDs, which we demonstrate for the up to the 4-state case.

In this work, we suggest using that the intrinsic uncertainty in the conduction paths of resonant tunnelling diodes can behave as a source of randomness that can be integrated into current electronics to produce on-chip true random number generators. The N-shaped I-V characteristic of RTDs results in a two-level random voltage output when driven with current pulse trains. Electrical characterisation and randomness testing of the devices was conducted in order to determine the validity of the true randomness assumption. Based on the results obtained for the single RTD case, we suggest the possibility of using multi-well devices to generate $\mathrm{N}$-state random switching devices for their use in random number generation or multi-valued logic devices.
\end{abstract}

Keywords: Resonant tunneling diode, random number generation, multi-valued logic, quantum well

\section{INTRODUCTION}

Random number generators (RNGs) are important in many diverse applications such as cryptography, simulation in the physical and social sciences, testing and gaming ${ }^{1}$. Many implementations rely on software- and hardware-based pseudo-random number generators, but true random numbers are required for sensitive applications like key generation in banking, defence or even social media. These requirements come from the fact that true random number generators, as opposed to their pseudo-random counterpart, are systems whose outputs cannot be determined even if their internal structure and response history are known ${ }^{1}$. True random numbers can be obtained from noise ${ }^{2}$, chaotic systems $^{3}$ and quantum phenomena ${ }^{4}$. In this work, we propose using resonant tunnelling diodes (RTD) as a practical, scalable source of randomness that employs quantum confinement. These devices operate at room temperature and are easy to integrate into current devices and microelectronic systems. The potential to epitaxially integrate single elements as RNGs into current technologies makes them resistant to frequency injection and biasing attacks, which affect state-of-the-art RNGs such as those based on free running oscillators ${ }^{5}$. This attack resistance can also be achieved using N-bit devices made up from multiple RTDs monolithically grown into a single structure ${ }^{6}$. This could enable their integration into small, low-power devices such as those used in the internet of things (IoT).

Resonant tunnelling diodes are the physical realisation of a semiconductor quantum well (QW) with finite rectangular barriers ${ }^{7,8}$. They normally consist of a thin, narrow band-gap semiconductor structure acting as a quantum well between two wide band-gap semiconductor tunnelling barriers ${ }^{9}{ }^{10}$. Beyond the tunnelling barriers, highly-doped regions of the narrow band-gap semiconductor act as an electron source/sink and are usually referred to as the emitter/collector regions, analogous to traditional bipolar transistors. Recently, resonant tunnelling devices using 
quantum dots ${ }^{11,} 12$, atomic-scale defects ${ }^{13}$, graphene ${ }^{14,15}$ and other two-dimensional materials ${ }^{16,17}$ have been demonstrated, and this has renewed the interest in investigating resonant tunnelling and its applications using new materials.

RTDs show the characteristic N-shaped I-V curve of negative differential resistance devices. A typical RTD characteristic is shown in Figure 1. The current obtained on the first slope of the curve arises due to the resonant tunnelling process ${ }^{7,18}$ that gives this device its name. Here, the bias voltage has shifted the first QW state between the emitter's Fermi level and the lower edge of its conduction band, facilitating the resonant tunnelling of electrons and allowing a reasonably high current to be measured. Once the confined level falls below the conduction band, a sudden drop in current can be observed as there are no more occupied states in the emitter aligned with the QW state. Further increase of the voltage leads to an increase in the current due to other conduction processes such as thermionic emission of hot electrons over the two tunnelling barriers ${ }^{18}$. The sudden current drop occurring between the two conduction regimes shows as a narrow resonance in the I-V characteristics. The bistability ${ }^{19}$ and fast switching characteristics ${ }^{20}$ emerging from the sharp NDR resonance of RTDs makes them promising candidates for applications in multi-valued logic circuits ${ }^{21}$, random-access memories ${ }^{22}$, multi-function logic gates ${ }^{23}$, chaotic signal generation ${ }^{3,24}$, ${ }^{25}$, single-photon switching ${ }^{26}$, unique device identification ${ }^{27}$ and terahertz oscillators ${ }^{28,29}$.

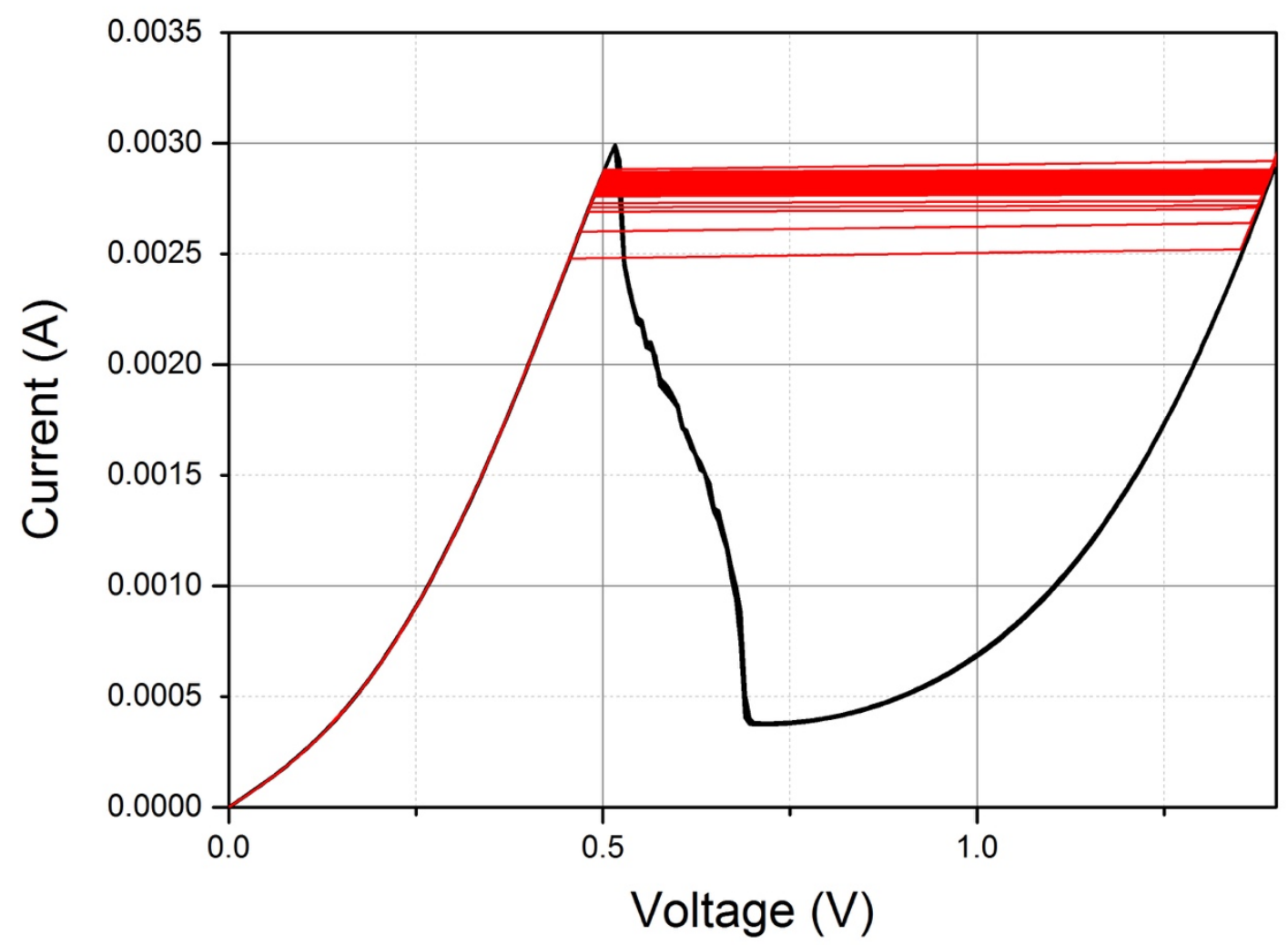

Figure 1. I-V characteristic of a RTD using a 10 voltage sweeps (black lines) and 100 current sweeps (red lines)

Due to their N-shaped NDR characteristics, the RTD's current is a single-valued function of voltage whereas the opposite is not true, as current values between those of the peak $\left(I_{p}\right)$ and the valley $\left(I_{v}\right)$ exhibit multiple voltage levels due to the different aforementioned conduction mechanisms. Using a current source in that range can result in two different scenarios: a low-resistance state (L) corresponding to the resonant tunnelling process, and a high-resistance 
state $(\mathrm{H})$ when transport of electrons is dominated by thermionic emission. Ramping up and down in current results in a hysteresis cycle $30-32$ between these two resistance states.

\section{METHODS}

The RTDs were fabricated from an InGaAs/AlAs double-barrier structure grown by Molecular Beam Epitaxy (MBE) on an InP substrate. To fabricate the RTDs, a top contact was first defined using conventional i-line optical lithography. A non-alloyed ohmic contact method was employed, where titanium $(50 \mathrm{~nm})$ and gold $(250 \mathrm{~nm})$ were deposited onto the surface of the highly doped cap layer by thermal evaporation. The top metal itself acted as a hard mask for a subsequent mesa etch. A reactive-ion etching (RIE) process using a mixture of methane $\left(\mathrm{CH}_{4}\right)$ and hydrogen $\left(\mathrm{H}_{2}\right)$, with an etch rate of $21 \mathrm{~nm} /$ minute, was implemented in order to produce anisotropic side-walls to the bottom contact layer, in preparation for the bottom metal contact deposition. Just before the bottom metal contact process took place, a non-selective orthophosphoric-based $\left(\mathrm{H}_{2} \mathrm{O}: \mathrm{H}_{3} \mathrm{PO}_{4}: \mathrm{H}_{2} \mathrm{O}_{2}=50: 3: 1\right)$ wet-etch with a etch rate of 90 nm/minute was used to etch away $200 \mathrm{~nm}$ of epilayers down to the surface of the InP to completely isolate neighbouring devices. The 5 minutes wet-etch also simultaneously provided the necessary undercut for the air-bridge formation. Finally, the bottom ohmic contact was formed by thermal evaporation of Ti/Au $(50 \mathrm{~nm} / 500 \mathrm{~nm})$. The exact details of the fabrication process are described elsewere ${ }^{9}$.

The fabricated chips include devices with mesa sizes of $4 \mu \mathrm{m}^{2}, 9 \mu \mathrm{m}^{2}, 16 \mu \mathrm{m}^{2}, 25 \mu \mathrm{m}^{2}$, and $36 \mu \mathrm{m}^{2}$; their I-V characteristics scale with size. The measurements shown in this work were performed for all the different device sizes and show no qualitative differences except for the current scaling for different device sizes. In the case of devices connected in series, we chose those which show the least differences in their I-V curves in order to demonstrate the principle, this corresponded to two pairs of RTDs of $4 \mu \mathrm{m}^{2}$ and $25 \mu \mathrm{m}^{2}$.

The devices were ball-bonded to a ceramic chip carrier using a TPT HB05 Ultrasonic wire bonder with a $25 \mu \mathrm{m}-$ diameter gold wire. Connections between RTDs and to the source and measurement equipment was done using standard copper wires and connectors.

All measurements were taken at room temperature. The voltage sweeps, current sweeps and DC current pulse trains were performed using a Keithley 2400 source measure unit. The time monitoring was performed with a mixed signal Tekronix DPO2100 oscilloscope.

\section{RESULTS AND DISCUSSION}

Our previous results showed that the switching threshold from one state to the other is not a fixed value but is statistically distributed near the resonance ${ }^{32}$ (Figure 1). As expected, currents above $\mathrm{I}_{\mathrm{p}}$ will always send the system to the high resistance state as the low-resistance branch does not reach that current range, and setting the current below $I_{v}$ will always give the low resistance state for similar reasons. Working between these limits (from $I_{v}$ to $I_{p}$ ) results in a non-deterministic switching behaviour, as the state change form $\mathrm{L}$ to $\mathrm{H}$ happens at different values each time following a certain probability distribution.

In order to characterise this random switching we use two different approaches. Full characterization is carried out by performing current sweeps as shown in Figure 1. In order to demonstrate the operation of RTDs as sources of randomness, we studied their response to square-wave current pulse trains of varying widths. This way we can produce streams of random states based on the inherent randomness of the switching process (Figure 2). The state was measured as the voltage drop across the RTD for a fixed current at the end of the pulse, where L corresponds to a low voltage drop and $\mathrm{H}$ to a high voltage drop at the end of the pulse (Figure 2-a). The responses to three trains of 7500 pulses at a fixed driving current, with pulse widths from $1 \mathrm{~ms}$ to $300 \mathrm{~ms}$, are plotted as a 2D map in Figure 2-b. We observed that the $\mathrm{L} / \mathrm{H}$ ratio of the measured state depends both on the current level and the pulse width. Resonant tunnelling is a time-dependent process ${ }^{7}$ and so is the switching between different states in these devices; the longer the source is on, the higher the chance of getting the high-resistance state at the end of the pulse. This time dependence was characterised by monitoring the voltage drop in the RTD with a high-resolution oscilloscope whilst sending consecutive current pulses. The waveforms are shown in Figure 2-a and clearly show that the time elapsed before each 
$\mathrm{L}$ to $\mathrm{H}$ switch is irregular, which makes the pulse width an important parameter in determining the characteristics of the random response.
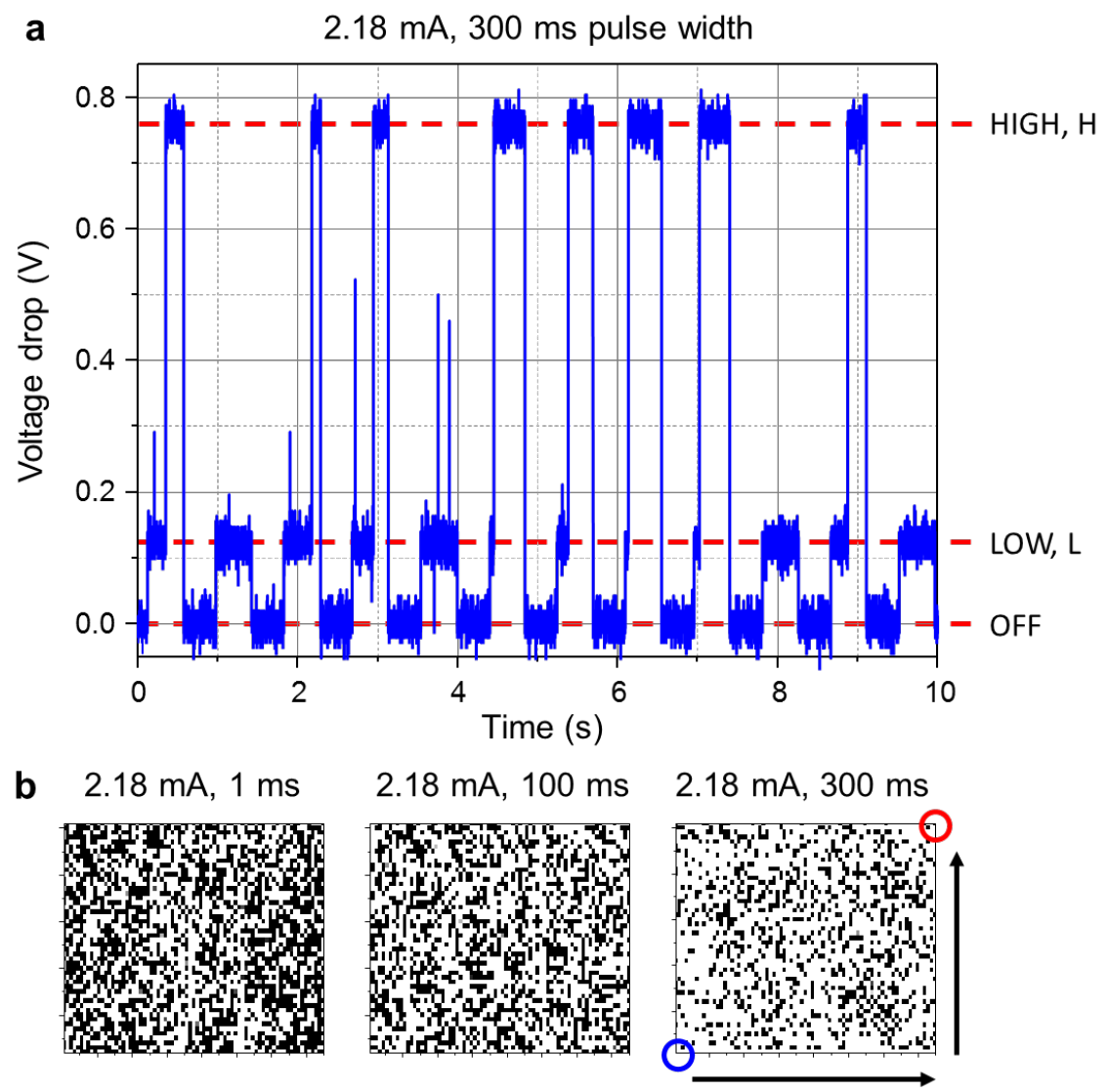

Figure 2. (a) Oscilloscope trace of the voltage dropped over a $25 \mu \mathrm{m} 2$ RTD (RTD 1 in Figure 5-a) when probing it with $2.18 \mathrm{~mA}$ square pulses with a repetition rate of $600 \mathrm{~ms}$ and $50 \%$ duty cycle (300 ms pulse width). The voltage levels corresponding to the high- and low-resistance states along with the off state are marked by the right axis. (b) Twodimensional representation of the measured state for 7500 pulses (50x150), wrapped around into a rectangle, with different pulse widths as labelled. The consecutive measurements are displayed left to right and bottom to top as shown by the arrows in the last panel. Black pixels represent the low resistance state $(\mathrm{V} \leq 0.4 \mathrm{~V})$ and white the high resistance state $(\mathrm{V}>0.4 \mathrm{~V})$. The response to the first pulse is circled in blue and that of the last in red. The series of measurements with longer pulse widths show an increased chance to be found in the high resistance state.

The I-V characteristic of two similar RTDs connected in series shows two consecutive resonances ${ }^{33}$, as illustrated in Figure 3-c. Considering the results shown for a single RTD and the I-V characteristics of combined RTDs, there is a current range at which it is possible to have a three-state system due to the three-valued nature of voltage as a function of current. For these experiments we have chosen RTDs with similar characteristics, such that the I-V curves are almost identical; albeit with small differences in the peak positions (see Figure 3-a). The uniformity in characteristics between the two diodes corresponds to better than 0.02ML accuracy in barrier thickness, demonstrating the outstanding control over tunnelling devices permitted by molecular beam epitaxy ${ }^{34}$. In this case, we can consider that the voltage drop across each device is approximately half the voltage applied to the whole system at a given time, so the first current drop corresponds to the device with a lower $V_{p}$ (smaller $I_{p}$ ) switching from resonant tunnelling to thermionic emission. Likewise, the second resonance corresponds to the same process in the device with a higher $V_{p}$ $\left(\mathrm{I}_{\mathrm{p}}\right.$ ). Considering the results shown above for a single RTD, we denote the three possible states as LL (both RTDs are in the $\mathrm{L}$ state), $\mathrm{LH}$ (one is $\mathrm{L}$ and the other $\mathrm{H}$ ), and HH (both RTDs are in the H state). 
a

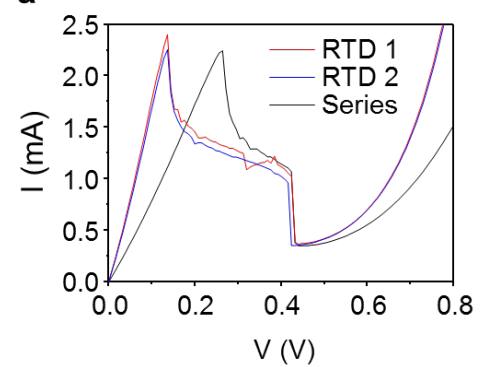

b

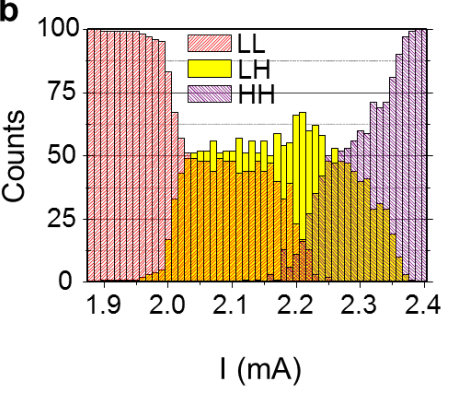

C

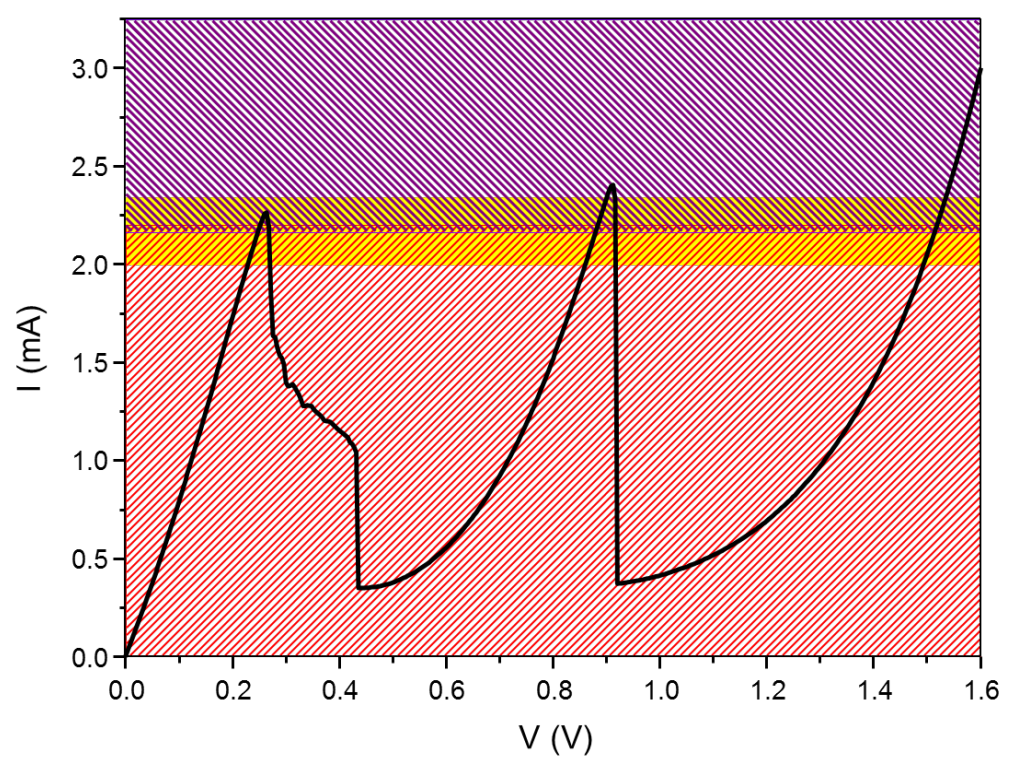

Figure 3. (a) I-V curves of two similar $25 \mu \mathrm{m} 2$ RTDs; RTD1 (red line), RTD2 (blue line) and the first peak of their series response (black line). (b) Histogram showing the probability of getting one of the three different states, namely LL (red columns), LH (yellow columns) or HH (purple columns), at different currents with a pulse width of $100 \mathrm{~ms}$. The measurements were performed with $0.01 \mathrm{~mA}$ steps, 100 pulses per step. (c) Full I-V curve of the two devices connected in series showing the two resonances and the last high-resistance slope. The shaded background regions correspond to the current ranges at which each of the states can occur with a probability higher than $20 \%$ according to the data in (b).

Performing current pulse train experiments, we measured the probabilities of obtaining each of the three possible states. Figure 3-b shows a histogram of one of these experiments. As one would expect, for currents far from the peak we obtain a majority of LL, while for currents close to the resonance most of the responses are HH. In the intermediate region, we get LL-LH, LL-LH-HH and LH-HH. The time-dependent voltage traces in Figure 4 show the response of the combined devices as measured with an oscilloscope. The average time between state changes is reduced for increasing current. At currents near the resonance there is a higher probability for the system to switch to $\mathrm{HH}$, whilst this probability decreases when lower currents are used. This is demonstrated by the $24 \mathrm{HH}$ peaks in $50 \mathrm{~s}$ from Figure 4-a $(2.35 \mathrm{~mA})$ and only $2 \mathrm{HH}$ peaks in Figure 4-b $(2.30 \mathrm{~mA})$ in the same time period. Experiments performed at lower currents, far from $I_{p}$, show that there is always a finite probability to measure the HH value, even when it is statistically improbable (Figure 4-c). 

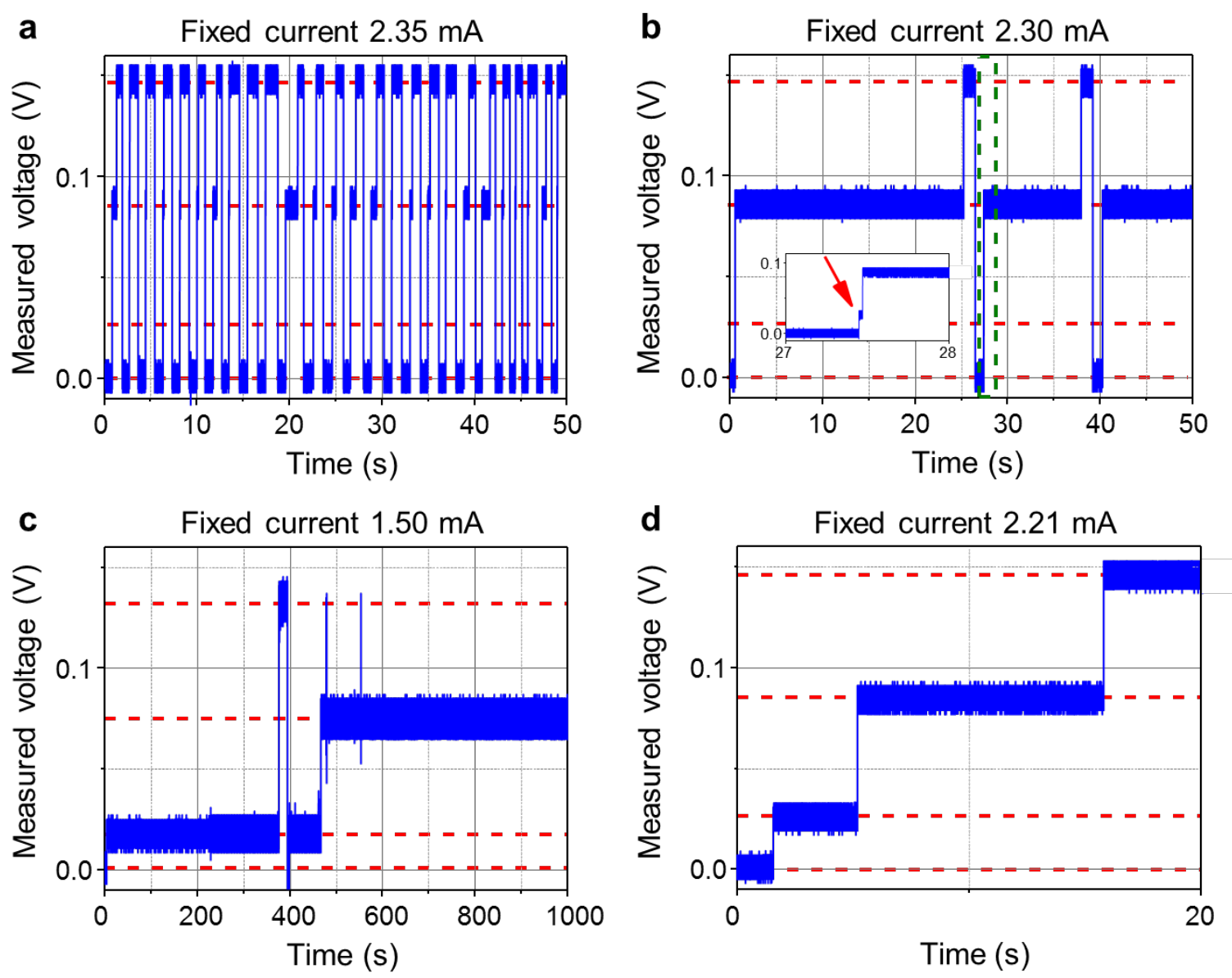

Figure 4. Time-dependent voltage traces of the same device excited at four different DC currents: (a) $2.35 \mathrm{~mA}, 50 \mathrm{~s}$, (b) $2.30 \mathrm{~mA}, 50 \mathrm{~s}$, (c) $1.50 \mathrm{~mA}, 1000 \mathrm{~s}$, (d) $2.21 \mathrm{~mA}, 20 \mathrm{~s}$. The blue lines correspond to the voltage drop across the two RTDs. The four stable levels (OFF, LL, LH and HH for increasing voltages) are marked in each panel with red dashed lines. In (a), (b) and (c), whenever the system jumps to HH the source is turned off and on again in order to reset the system. The inset in (b) is a $1 \mathrm{~s} \mathrm{zoom}$ to the green dashed rectangle showing how the system passes through the LL state for a brief period of time (red arrow) before switching to LH. Far from resonance (c), there is an increased stability where the state is less likely to change over time, evidenced by the LL and LH states remaining unchanged for 4 minutes and 9 minutes respectively. However, there is still a finite probability of switching to the $\mathrm{HH}$ state, as observed at approximately $400 \mathrm{~s}$.

Connecting three similar RTDs in series, such as those shown in Figure 5-a, results in a 3-peak system that can be used as a 4-state device, as the combined I-V curve presents four stable slopes. The characteristic curve of one of these systems is shown in Figure 5-b. Akin to the 2- and 3-state cases, the thresholds for each of the state changes follows a specific probability distribution. The exact probability distribution for each level depends on the differences between the individual RTDs. The device shown in Figure 5 is comprised of three RTDs with similar characteristics (i.e. $\mathrm{I}_{\mathrm{p}}$, slope) and results in a broad range of currents that can output the four different states. If the RTDs were dissimilar, this range would be reduced due to the misalignment of the peak and valley currents of the different peaks. Scaling to $\mathrm{N}$-bit devices is trivial as it only requires the series connection of additional RTDs, although for practical implementation, there is a limit to the number of devices that can be connected this way due to voltage and power requirements. 

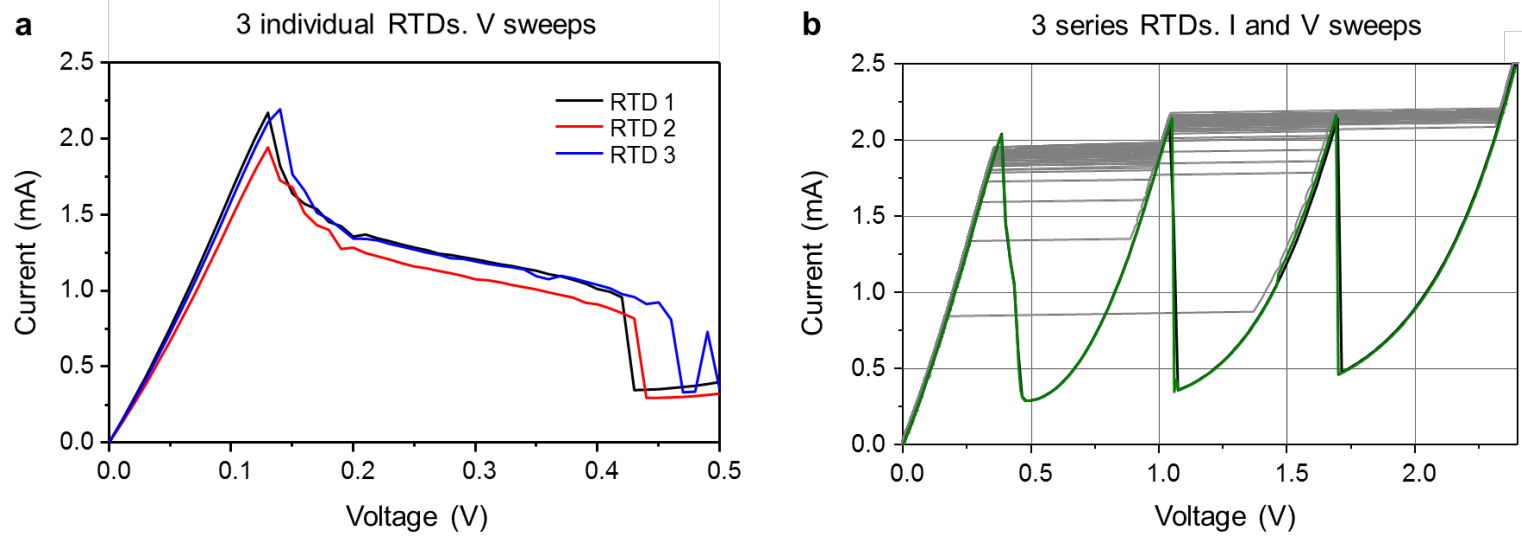

Figure 5. (a) I-V curves of three $25 \mu \mathrm{m}^{2}$ RTDs when driven by a voltage sweep. (b) I-V characteristic of the three devices in (a) connected in series showing a voltage sweep (green curve) and 120 current sweeps (grey lines), showing the random switching behaviour between the four states corresponding to the four stable points on the curve.

\section{CONCLUSIONS}

We have demonstrated how the hysteretic behaviour of resonant tunnelling diodes can be exploited in order to get a non-deterministic two-level state machine within the $I_{v}$ to $I_{p}$ current range. This behaviour can be extended to a series of connected RTDs, to produce N-bit devices as we have shown for the 3-, and 4-state cases. The multiple resonances of combined RTDs create a number of different stable states each with their own switching probability distribution. This allows random voltage streams to be achieved by measuring the device with current pulse trains. These quantumbased random-output devices can be easily integrated into microelectronic systems and operate at room temperature, making them useful for different applications. The two-state random response devices can be used to produce streams of random bits which can then be used for cryptography. The ability to tune the ratio between the two possible states following a non-uniform probability distribution can prove useful for simulation purposes in physical or social sciences. The $\mathrm{N}$-state devices driven in the non-deterministic range can be also used for random number generation, but further applications can be devised in multi-valued logic circuits or for the random routing of signals. In addition to true random number generation ${ }^{2,435}$, and due to their multi-valued hysteretic behaviour, these devices show great potential for novel applications such as elements for neural networks ${ }^{36,37}$ and multi-valued logic systems ${ }^{21}$.

\section{REFERENCES}

[1] Ç. K. Koç, and M. Stipcevic, [True random number generators] Springer, New York(2014).

[2] D. G. Marangon, G. Vallone, and P. Villoresi, "Random bits, true and unbiased, from atmospheric turbulence," Sci Rep, 4, 5490 (2014).

[3] K. Maezawa, Y. Kawano, Y. Ohno et al., "Direct Observation of High-Frequency Chaos Signals from the Resonant Tunneling Chaos Generator," Japanese Journal of Applied Physics, 43(8A), 5235-5238 (2004).

[4] X. Ma, X. Yuan, Z. Cao et al., "Quantum random number generation," npj Quantum Information, 2, 16021 (2016).

[5] A. T. Markettos, and S. W. Moore, "The Frequency Injection Attack on Ring-Oscillator-Based True Random Number Generators," Cryptographic Hardware and Embedded Systems - Ches 2009, Proceedings, 5747, 317-331 (2009).

[6] A. C. Seabaugh, Y. C. Kao, and H. T. Yuan, "Nine-state resonant tunneling diode memory," IEEE Electron Device Letters, 13(9), 479-481 (1992).

[7] B. Ricco, and M. Y. Azbel, "Physics of resonant tunneling. The one-dimensional double-barrier case," Physical Review B, 29(4), 1970-1981 (1984).

[8] L. L. Chang, L. Esaki, and R. Tsu, "Resonant tunneling in semiconductor double barriers," Applied Physics Letters, 24(12), 593-595 (1974). 
[9] M. A. M. Zawawi, K. W. Ian, J. Sexton et al., "Fabrication of Submicrometer InGaAs/AlAs Resonant Tunneling Diode Using a Trilayer Soft Reflow Technique With Excellent Scalability," Ieee Transactions on Electron Devices, 61(7), 2338-2342 (2014).

[10] D. J. Paul, P. See, R. Bates et al., "Si/SiGe electron resonant tunneling diodes with graded spacer wells," Applied Physics Letters, 78(26), 4184 (2001).

[11] G. Chen, G. Klimeck, S. Datta et al., "Resonant tunneling through quantum-dot arrays," Physical Review B, 50(11), 8035-8038 (1994).

[12] K. W. Lee, C. W. Jang, D. H. Shin et al., "Light-induced negative differential resistance in graphene/Siquantum-dot tunneling diodes," Sci Rep, 6, 30669 (2016).

[13] D. M. Di Paola, M. Kesaria, O. Makarovsky et al., "Resonant Zener tunnelling via zero-dimensional states in a narrow gap diode," Sci Rep, 6, 32039 (2016).

[14] L. Britnell, R. V. Gorbachev, A. K. Geim et al., "Resonant tunnelling and negative differential conductance in graphene transistors," Nat Commun, 4, 1794 (2013).

[15] A. Mishchenko, J. S. Tu, Y. Cao et al., "Twist-controlled resonant tunnelling in graphene/boron nitride/graphene heterostructures," Nat Nanotechnol, 9(10), 808-13 (2014).

[16] L. N. Nguyen, Y. W. Lan, J. H. Chen et al., "Resonant tunneling through discrete quantum states in stacked atomic-layered MoS2," Nano Lett, 14(5), 2381-6 (2014).

[17] K. A. Guerrero-Becerra, A. Tomadin, and M. Polini, "Resonant tunneling and the quasiparticle lifetime in graphene/boron nitride/graphene heterostructures," Physical Review B, 93(12), (2016).

[18] S. M. Sze, and K. K. Ng, [Physics of Semiconductor Devices] John Wiley \& Sons, Inc., New Jersey, $415-$ 465 (2006).

[19] F. W. Sheard, and G. A. Toombs, "Space-Charge Buildup and Bistability in Resonant-Tunneling DoubleBarrier Structures," Applied Physics Letters, 52(15), 1228-1230 (1988).

[20] N. C. Kluksdahl, A. M. Kriman, D. K. Ferry et al., "Transient Switching Behavior of the ResonantTunneling Diode," Ieee Electron Device Letters, 9(9), 457-459 (1988).

[21] Z. X. Yan, and M. J. Deen, "A New Resonant-Tunnel Diode-Based Multivalued Memory Circuit Using a Mesfet Depletion Load," Ieee Journal of Solid-State Circuits, 27(8), 1198-1202 (1992).

[22] G. Ternent, and D. J. Paul, "Si/SiGe Tunneling Static Random Access Memories," ECS Transactions, 50(9), 987-990 (2013).

[23] K. J. Chen, T. Akeyoshi, and K. Maezawa, "Monostable Bistable Transition Logic Elements (Mobiles) Based on Monolithic Integration of Resonant-Tunneling Diodes and Fets," Japanese Journal of Applied Physics Part 1-Regular Papers Brief Communications \& Review Papers, 34(2b), 1199-1203 (1995).

[24] Y. Kawano, S. Kishimoto, K. Maezawa et al., "Resonant tunneling chaos generator for high-speed/lowpower frequency divider," Japanese Journal of Applied Physics Part 2-Letters \& Express Letters, 38(11b), L1321-L1322 (1999).

[25] Y. Kawano, S. Kishimoto, K. Maezawa et al., "Robust operation of a novel frequency divider using resonant tunneling chaos circuit," Japanese Journal of Applied Physics Part 1-Regular Papers Short Notes \& Review Papers, 39(6a), 3334-3338 (2000).

[26] Q. Weng, Z. An, B. Zhang et al., "Quantum dot single-photon switches of resonant tunneling current for discriminating-photon-number detection," Sci Rep, 5, 9389 (2015).

[27] J. Roberts, I. E. Bagci, M. A. Zawawi et al., "Using Quantum Confinement to Uniquely Identify Devices," Sci Rep, 5, 16456 (2015).

[28] N. Orihashi, S. Hattori, S. Suzuki et al., "Experimental and Theoretical Characteristics of Sub-Terahertz and Terahertz Oscillations of Resonant Tunneling Diodes Integrated with Slot Antennas," Japanese Journal of Applied Physics, 44(11), 7809-7815 (2005).

[29] M. Asada, and S. Suzuki, "Theoretical analysis of external feedback effect on oscillation characteristics of resonant-tunneling-diode terahertz oscillators," Japanese Journal of Applied Physics, 54(7), 070309 (2015).

[30] S. E. Savel'ev, F. Marchesoni, and A. M. Bratkovsky, "Mesoscopic resistive switch: non-volatility, hysteresis and negative differential resistance," The European Physical Journal B, 86(12), (2013).

[31] D. B. Strukov, G. S. Snider, D. R. Stewart et al., "The missing memristor found," Nature, 453(7191), 80-3 (2008).

[32] R. Bernardo-Gavito, I. Ethem Bagci, J. Roberts et al., "Extracting random numbers from quantum tunnelling through a single diode," ArXiv, 1706.07348, (2017). 
[33] K. J. Gan, and Y. K. Su, "Improved circuit design of multipeak current-voltage characteristics based on resonant tunneling diodes," Japanese Journal of Applied Physics Part 1-Regular Papers Short Notes \& Review Papers, 36(10), 6280-6284 (1997).

[34] M. Missous, M. J. Kelly, and J. Sexton, "Extremely Uniform Tunnel Barriers for Low-Cost Device Manufacture," Ieee Electron Device Letters, 36(6), 543-545 (2015).

[35] M. Fujiwara, A. Waseda, R. Nojima et al., "Unbreakable distributed storage with quantum key distribution network and password-authenticated secret sharing," Sci Rep, 6, 28988 (2016).

[36] M. D. Pickett, G. Medeiros-Ribeiro, and R. S. Williams, "A scalable neuristor built with Mott memristors," Nat Mater, 12(2), 114-7 (2013).

[37] A. Serb, J. Bill, A. Khiat et al., "Unsupervised learning in probabilistic neural networks with multi-state metal-oxide memristive synapses," Nat Commun, 7, 12611 (2016). 